Reprod. Nutr. Dévelop., 1980, 20 (5 B), 1691-1694.

\title{
Étude préliminaire de l'effet d'une supplémentation en fer, cuivre, cobalt et zinc sur l'activité cellulolytique dans le cæcum du poney
}

par J. L. TISSERAND, F. BOULARD, Ph. DEPONGE

avec la collaboration technique de Catherine CORDELET

Laboratoire de Recherches de la Chaire de Zootechnie

ENSSAA-INRA, 26, Bd Dr Petitjean 21100 Dijon, France.

Summary. Preliminary study of the effect of increased amounts of iron, copper, cobalt and zinc on cellulolytic activity in the pony coecum.

We studied a caecum-cannulated pony fed hay ad libitium in order to determine the mineral elements likely to cause deficiencies which decrease microbial activity in the caecum.

During four successive 1-month periods, we observed the effects of adding $50 \mathrm{mg}$ of iron, $1 \mathrm{mg}$ of cobalt, $40 \mathrm{mg}$ of copper and $200 \mathrm{mg}$ of zinc, respectively. Except for the introduction of zinc, which greatly diminishes cellulolytic activity measured with the nylon bag technique, there was no significant modification in that activity as shown by $\mathrm{pH}, \mathrm{NH}_{3}$-nitrogen, total nitrogen and volatile fatty acid production (table 1 ).

It can be concluded that endogenous secretion in the pre-caecal part of the digestive system of the mineral elements used was sufficient to guarantee good symbiosis between the microbial population of the caecum and the organism.

\section{Introduction.}

La digestion microbienne dans le gros intestin des équidés est un élément déterminant pour l'utilisation des fourrages chez cette espèce (Alexander, 1972 ; Hintz et al., 1971 ; Robinson et Slade, 1974 ; Tisserand, 1979 ; Wolter, 1975).

La présence d'éléments minéraux et particulièrement d'oligo-éléments qui jouent un rôle déterminant pour la croissance de la population du rumen (Durand ef Kawashima, 1979) est susceptible de modifier l'activité microbienne lors de la digestion chez le cheval. Ce facteur est d'autant plus important que les échanges entre le contenu digestif et le sang dans la fraction pré-cæcale de l'appareil digestif des équidés peuvent induire des carences réduisant la croissance microbienne dans le cæcum de ces animaux.

Dans le but de déterminer les éléments minéraux pouvant se trouver en quantité insuffisante dans le cæcum ainsi que les conditions d'une expérimentation fiable nous avons étudié les effets éventuels de l'introduction de fer, cuivre, cobalt et zinc directement dans le cæcum du poney. 


\section{Techniques expérimentales et matériel utilisé.}

L'animal. - L'étude a été conduite sur un seul poney mâle Fanfan $(F)$ pesant $160 \mathrm{~kg}$ âgé de 7 ans et porteur de 2 canules, l'une du cæcum, l'autre du côlon ventral posées par M. Candau et C. Masson (Tisserand et al., 1977).

Le régime alimentaire. - Durant toute la durée de l'expérience, le sujet expérimenté reçoit du foin de pré distribué à volonté. Ce fourrage a la composition moyenne suivante par rapport à la matière sèche : 7,1 p. 100 de matière minérale, $35 \mathrm{p}$. $100 \mathrm{de}$ cellulose, 7,1 p. 100 de matières azotées totales, 1,8 g de phosphore, $4,4 \mathrm{~g}$ de calcium, $4 \mathrm{mg}$ de cuivre, $28 \mathrm{mg}$ de zinc et $344 \mathrm{mg}$ de fer.

L'eau de boisson est laissée à la libre disposition de l'animal. Chaque matin avant le repas et durant 4 périodes expérimentales successives d'un mois chacune, $100 \mathrm{~cm}^{3}$ d'eau distillée sont introduits directement dans le cæcum à travers la canule renfermant :

- 1 re période : $50 \mathrm{mg}$ de fer,

- $2^{\mathrm{e}}$ période : $1 \mathrm{mg}$ de cobalt,

- $3^{\text {e }}$ période : $40 \mathrm{mg}$ de cuivre,

- 4e période : $200 \mathrm{mg}$ de zinc.

Chaque séquence de deux périodes expérimentales est encadrée par deux périodes témoins avec addition de $100 \mathrm{~cm}^{3}$ d'eau distillée afin d'accoutumer les animaux à l'addition de liquide directement dans le cæcum.

Mesures effectuées. - Au cours des dix derniers jours de chaque période, l'activité cellulolytique dans le cæcum est évaluée par la technique des sacs de nylon à l'aide de deux fois deux sacs contenant $1 \mathrm{~g}$ de foin de pré et séjournant $24 \mathrm{~h}$ dans le cæcum. Le dosage de la cellulose est effectué à l'aide de la méthode Van Soest (Boulard, 1979 ; Deponge, 1979).

De même 3 séries de 3 prélèvements de contenu du cæcum sont réalisées à 4 jours d'intervalles, à $10 \mathrm{~h}, 14 \mathrm{~h}$ et $17 \mathrm{~h} 30$. Chaque fois $100 \mathrm{~cm}^{3}$ environ du contenu de cæcum est obtenu par aspiration à l'aide d'une sonde en matière plastique enveloppée dans un sac de toile de nylon pour éviter de blesser l'animal (Tisserand et Masson, 1976).

Sur ces échantillons les déterminations suivantes sont systématiquement effectuées :

- mesure du $\mathrm{pH}$;

- dosage de l'azote ammoniacal par la méthode de Conway ;

- dosage de l'azote total par la méthode Kjeldhal ;

- séparation et dosage des acides gras volatils (contenant 2 à 5 carbones) par chromatographie en phase gazeuse (support chromosorb W 80-100 m phase stationnaire NPGA 25 p. 100 + acide phosphorique 2 p. 100).

Des moyennes sont établies pour chaque journée de prélèvement.

\section{Résultats.}

Les résultats moyens de chaque période sont rassemblés dans le tableau 1. 
TABLEAU 1

Effet de l'addition de fer, cobalt, cuivre ef zinc sur l'activité microbienne dans le cæcum du poney

\begin{tabular}{|c|c|c|c|c|c|c|c|c|}
\hline & Témoin & Fer & Cobalt & Témoin & Témoin & Cuivre & Zinc & Témoin \\
\hline $\begin{array}{l}\text { Activité cellulolytique. } \\
\text { pH. } \ldots \ldots \ldots \ldots \ldots \ldots \ldots \\
\mathrm{NH}_{3} / \mathrm{N}_{\mathrm{T}} \ldots \ldots \ldots \ldots \ldots \\
\text { Acides gras volatils }\end{array}$ & $\begin{array}{r}43,55 \\
7,64 \\
4,71\end{array}$ & $\begin{array}{r}45,25 \\
7,15 \\
4,22\end{array}$ & $\begin{array}{r}31,82 \\
7,06 \\
5,15\end{array}$ & $\begin{array}{r}31,65 \\
7,16 \\
5,31\end{array}$ & $\begin{array}{r}28,06 \\
7,52 \\
6,01\end{array}$ & $\begin{array}{r}29,29 \\
7,59 \\
6,21\end{array}$ & $\begin{array}{r}13,92 \\
7,35 \\
4,25\end{array}$ & $\begin{array}{r}33,32 \\
7,35 \\
4,38\end{array}$ \\
\hline $\begin{array}{l}\text { totaux (mmoles/l) } \ldots \\
\text { A. acétique (p. 100) } \ldots \\
\text { A. propionique }(p .100) \\
\text { A. butyrique }(\text { p. 100) . . }\end{array}$ & $\begin{array}{r}45,05 \\
75,86 \\
18,42 \\
4,33\end{array}$ & $\begin{array}{r}46,98 \\
73,13 \\
19,81 \\
5,49\end{array}$ & $\begin{array}{r}48,70 \\
72,82 \\
19,61 \\
6,78\end{array}$ & $\begin{array}{r}50,32 \\
71,32 \\
20,69 \\
6,54\end{array}$ & $\begin{array}{r}42,68 \\
72,32 \\
19,23 \\
6,53\end{array}$ & $\begin{array}{r}37,36 \\
68,98 \\
23,32 \\
5,91\end{array}$ & $\begin{array}{r}41,96 \\
66,44 \\
25,30 \\
6,58\end{array}$ & $\begin{array}{r}35,37 \\
67,76 \\
23,59 \\
6,55\end{array}$ \\
\hline
\end{tabular}

Par rapport à la première période témoin l'addition de fer ne semble pas modifier ni l'activité cellulolytique, ni les données biochimiques du contenu du cæcum à l'exception d'une légère diminution $\mathrm{du} \mathrm{pH}$ et du pourcentage d'azote ammoniacal par rapport à l'azote total.

Le passage de l'additif fer au cobalt se traduit par une diminution sensible de l'activité cellulolytique et une légère augmentation de la concentration en acides gras volatils totaux et du pourcentage molaire d'acide butyrique. Toutefois la comparaison à la période témoin qui suił ne permet pas de confirmer l'hypothèse d'un effet spécifique de l'addition de cobalt sur l'activité microbienne du cæcum.

L'introduction de cuivre ne modifie pas l'activité cellulolytique dans le cæcum mais $y$ entraine une baisse de la concentration en acides gras volatils totaux avec diminution du pourcentage molaire de l'acide acétique au profit de l'acide propionique. Par contre, l'introduction de zinc dans le cæcum entraîne une baisse importante de l'activité cellulolytique, sans modification de la concentration en acides gras volatils totaux, mais avec une diminution du pourcentage molaire d'acide acétique.

\section{Discussion et conclusion.}

II convient d'abord de souligner qu'il est impossible de tirer des conclusions d'observations faites sur un seul sujet, mais cette étude a un caractère indicatif et semble aussi fiable que des observations effectuées uniquement in vitro. II semble qu'indépendamment de l'effet de différents additifs il existe une évolution dans le temps de l'activité cellulolytique du contenu du cæcum dont il s'avère difficile de chiffrer l'importance.

En conséquence il paraît prudent de se limiter à observer que dans les conditions de notre essai le fer, le cobalt et le cuivre ne constituent vraisemblablement pas des facteurs limitants de l'activité microbienne dans le cæcum du poney. Pour le dernier élément, nos observations sont en accord avec les travaux de Hubbert. Cheng et Burroughs (1958) ef al., Martinez et Church (1970).

Par contre l'addition de zinc entraîne un effet inhibiteur de la cellulolyse déjà mis en évidence in vitro chez le ruminant par Bonhomme et al. (1979), tout en diminuant la production d'acides gras ef en particulier d'acide propionique, ce qui avait été mis en évidence antérieurement par Uesaka, Kawashima et Zembayashi (1966), également in vitro et sur ruminants. 
Malgré le fait que la solubilité des divers éléments minéraux apparaisse relativement faible dans le cæcum, il semble que les sécrétions endogènes des éléments minéraux expérimentés dans la fraction précæcale de l'appareil digestif soient suffisantes pour assurer une bonne symbiose entre la population microbienne du cæcum ef l'organisme de l'animal hôte.

D'autres études en cours sont toutefois nécessaires avec plusieurs sujets pour déterminer avec plus de sûreté les additifs minéraux susceptibles de stimuler la croissance microbienne dans le cæcum des équidés.

Journées Ingestion-Digestion-Absorption de l'Association française de Nutrition, Paris, 15-16 novembre 1979.

Remerciements. - Nous tenons à remercier Michèle Durand, Maître de Recherches à I'INRA et le personnel de la Station de Recherches de Nutrition de Jouy-en-Josas pour les analyses d'oligo-éléments effectuées au cours de cette étude.

Nos remerciements vont également à $M$. Candau, Maître de Conférences à I'E.N.S.A. de Toulouse, qui a pratiqué la fistulation du sujet expérimenté.

\section{Références}

ALEXANDER F., 1972. Some functions of the large intestine of the horse. Quart. J. exp. Physiol., $37,205$. BONHOMME A., DURAND M., DUMAY C., BEAUMATIN P., 1979. Elude in vitro du comportement des populations microbiennes du rumen en présence de zinc sous forme de sulfate. Ann. Biol. anim. Bioch. Biophys., 19, 937-942.

BOULARD F., 1979. Contribution à l'éfude de l'activité microbienne dans le gros infestin chez le poney. Essai de stimulation par addition de fer et de cobalt dans le cæcum. Mém. Fin d'études, E.S.A. Angers.

DEPONGE Ph., 1979. Contribution à l'étude de l'activité microbienne dans le gros intestin chez le poney. Essai de stimulation par infusion des deux éléments minéraux cuivre et zinc dans le cæcum. Mém. Fin d'études, E.N.I.T.A. Quetigny.

DURAND M., KAWASHIMA R., 1979. Influence of minerals in rumen microbial digestion. Vih int. Symp. Physiology of ruminants, Clermont-Ferrand.

HINTZ H. F., HOGUE D. E., WALKER E. F., LOWE J. E., SCHRYVER H. F., 1971. Apparent digestion in various segments of digestion tract of ponies fed diets with varying roughage grain ratios. $J$. anim. Sci., 32, 235-248.

HUBBERT F. Jr., CHENG E., BURROUGHS W., 1958. Mineral requirement of rumen microorganisms for cellulose digestion in vitro. J. anim. Sci., 17, 559-568.

MARTINEZ A., CHURCH D. C., 1970. Effect of various mineral elements on in vitro rumen cellulose digestion. J. anim. Sci., 31, 982-990.

ROBINSON D. W., SLADE L. M., 1974. The current statuts of knowledge on the nutrition of equine. J. anim. Sci., 39, 1045-1066.

TISSERAND J. L., MASSON C., 1976. Effet de différents régimes sur l'activité biochimique dans le gros intestin du poney. 27e Réun. annu. Féd. eur. Zootech., Zürich, 23-26 août.

TISSERAND J. L., MASSON C., OTTIN PECCHIO M., CREUSOT A., 1977. Mesure du pH et de la concentration en AGV dans le cæcum ef le côlon du poney. Ann. Biol. anim. Bioch. Biophys., 17, 533-537.

TISSERAND J. L., 1979. L'alimentation pratique du cheval. 1 vol. 87 pp., Ed. A. Lesson. Diff. Maloine S.A. Paris.

UESAKA J., KAWASHIMA R., ZEMBAYASHIM., 1966. In DURAND M. et KAWASHIMA R., 1979. WOLTER R., 1975. L'alimentation du cheval. $2^{\mathrm{e}}$ éd., 1 vol., 180 pp. Vigot Frères, Paris. 